\title{
Effect of chemical vapor deposition parameters on the diameter of multi-walled carbon nanotubes
}

\author{
Sivamaran Venkatesan ${ }^{1}$ (i) $\cdot$ Balasubramanian Visvalingam ${ }^{1} \cdot$ Gopalakrishnan Mannathusamy $^{2}$. \\ Viswabaskaran Viswanathan ${ }^{3} \cdot$ A. Gourav Rao ${ }^{4}$
}

Received: 29 July 2018 / Accepted: 18 October 2018 / Published online: 1 November 2018

(c) The Author(s) 2018

\begin{abstract}
The multi-walled carbon nanotubes (MWCNTs) with controlled diameter distribution are useful in the fabrication of composite materials as reinforcement, due to their superior strength and toughness. Chemical vapor deposition (CVD) is a viable process to synthesize MWCNTs. In this investigation, an attempt has been made to study the effect of CVD process parameters (reaction temperature, flow rate of precursor gas, process time) on the mean diameter of MWCNTs. The MWCNTs of controlled diameter distribution was selectively grown on $\mathrm{NiO}$ catalyst supported by $\mathrm{Al}_{2} \mathrm{O}_{3}$ nano particles. The argon and acetylene were used as carrier and carbon precursor gas, respectively. The catalyst and carbon samples were characterized using field emission scanning electron microscopy, high-resolution transmission electron microscopy, Raman spectroscopy and thermogravimetric/differential thermal analysis. From this investigation, it is understood that the increase in reaction temperature and flow rate of precursor gas increased the mean diameter of MWCNTs but increase in process time decreased the diameter. The diameter distribution and quality of MWCNTs are strongly influenced by the diameter of the catalyst particles.
\end{abstract}

Keywords Nanotechnology $\cdot$ Carbon nanotubes $\cdot$ Chemical vapor deposition $\cdot$ Mean diameter

Sivamaran Venkatesan

sivamaranv@gmail.com

Balasubramanian Visvalingam

visvabalu@yahoo.com

Gopalakrishnan Mannathusamy

mgkrishnan61@gmail.com

Viswabaskaran Viswanathan

vbceramic@yahoo.com

A. Gourav Rao

gouravdrdo@gmail.com

1 Centre for Materials Joining and Research (CEMAJOR), Department of Manufacturing Engineering,

Annamalai University, Annamalai Nagar (P.O), Chidambaram, Cuddalore District 608 002, Tamil Nadu, India

2 Department of Chemistry, Annamalai University, Annamalai Nagar (P.O), Chidambaram, Cuddalore District 608 002, Tamil Nadu, India

3 VB Ceramic Research Centre (VBCRC), 27A, 14th Link Road, 3rd Cross, Nehru Nagar Industrial Estate, Kottivakkam, Chennai 600 041, India

4 Naval Materials Research Laboratory (NMRL), Shil Badlapur Rd., MIDC Area, District Thane, Ambernath, Maharashtra, 421506, India

\section{Introduction}

The significance of carbon nanotubes (CNTs) in the field of nanotechnology was noticed abundantly by the researchers, after the tubular needle growth model, proposed by the Ijima in 1991 [1]. The growth model of CNTs was proposed to be, the rolling up graphene-hexagon sheet into a cylinder shape to form a tubular structure. The CNTs growth model possess the similar way of the bacon's scroll model [1]. CNTs are costly and highly demanded nanomaterial around the world, due to their excellent electrical conductivity [2], tensile strength [3] and thermal conductivity. An elastic modulus of multi-walled carbon nanotubes (MWCNTs) is tenfold higher than the any commercial, industrial fiber [4].

To use CNTs in many applications, it is indeed to produce these nanomaterials with highly precise structure and in large scale at an affordable cost. The CNTs were synthesized by various methods, mainly: arc discharge [5], laser ablation [6] and chemical vapor deposition (CVD) [7, 8]. As compared to other methods, CVD is a simple and economical technique for the growth of CNTs at low temperature and ambient pressure [9]. The required structure and 
morphology of CNTs were achieved by a set of controlled variables $[10,11]$.

Nickel oxide (NiO) was specifically selected as the catalyst material, due to its strong interaction with the outer graphitic plane of CNTs than some other metal oxide catalyst like copper $(\mathrm{Cu})$ [12-14]. $\mathrm{NiO}$ is supported by alumina $\left(\mathrm{Al}_{2} \mathrm{O}_{3}\right)$ because of its higher activity and its support to the metal catalyst in higher temperature for the growth of MWCNTs [15]. Acetylene gas seems to be favoring the growth of CNTs since it contains smaller carbon atoms [16].

CVD process parameters were varied to control the growth of CNTs, such as effect of precursor gas flow rate [17], carrier gas flow rate [18], process time [19], and reaction temperature [20]. Besides, only few researchers have investigated on the growth of CNTs by CVD process. No systematic study has been reported so far to quantitatively assess the effect of CVD parameters on mean diameter of MWCNTs. Therefore, this investigation was taken up to study the effect of CVD parameters such as reaction temperature, flow rate of precursor gas and reaction time on the mean diameter of MWCNTs.

\section{Experimental}

To achieve the desired objective, the present investigation was planned in the following sequence.

(i) Identify the important CVD process parameters and their feasible working limits

(ii) Prepare the suitable catalyst

(iii) Synthesize CNTs by CVD process

(iv) Purify CNTs by wet chemistry and filtering methods (v) Characterize MWCNTs by FESEM, HRTEM, TGA/ DTA, and Raman spectroscopy analysis

(vi) Analyze the results to optimize appropriate CVD process parameters to obtain MWCNTs with equal diameter distribution with defectless structure

\section{Identify the important CVD process parameters and their limits}

From the literature [21-24] review, the predominant CVD parameters which control the morphology and diameter of MWCNTs were identified. They are (a) reaction temperature (b) flow rate of precursor gas (c) process time. Trial experiments were conducted initially and $\mathrm{NiO}$ was used as the transition metal catalyst and it was supported by $\mathrm{Al}_{2} \mathrm{O}_{3}$. Ten milligrams of catalyst was loaded into the CVD chamber and it was maintained constant for all the experiments. The morphology of the $\mathrm{NiO} / \mathrm{Al}_{2} \mathrm{O}_{3}$ catalyst was spherical in shape (Fig. 1a). From the trial experiments, the following observations were made:

(i) If the reaction temperature was below $700{ }^{\circ} \mathrm{C}$, the growth of CNTs was affected due to the lesser active sites on the surface area of the catalyst. If the reaction temperature was greater than $1000^{\circ} \mathrm{C}$, the deposition of amorphous carbon and disoriented CNTs were observed, due to self-diffusion of acetylene towards the catalyst and also the over agglomeration of catalyst particles.

(ii) If the precursor gas flow rate was less than $100 \mathrm{ml} \mathrm{min}^{-1}$, there was no tubular morphology in the samples, due to insufficient diffusion of carbon atoms to the catalyst particles. If the precursor flow rate was more than $180 \mathrm{ml} \mathrm{min}^{-1}$, the highest number of tubular CNTs were found, but the amorphous
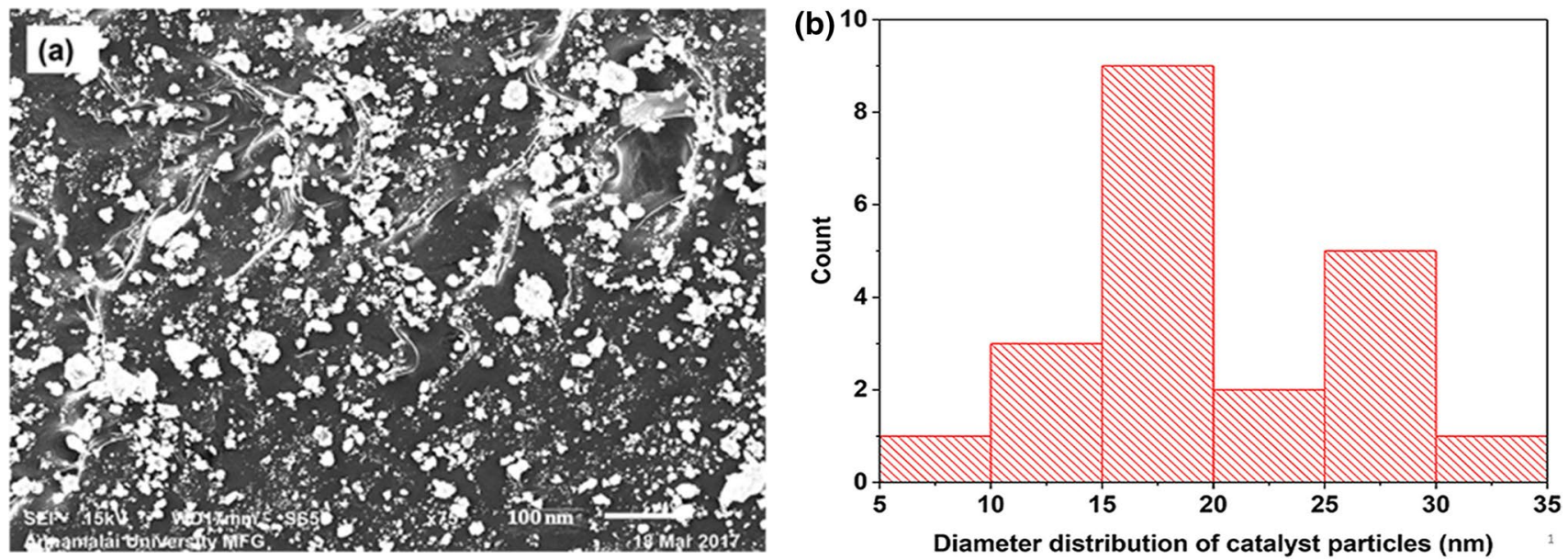

Fig. 1 a Morphology of $\mathrm{NiO} / \mathrm{Al}_{2} \mathrm{O}_{3}$ powder catalyst, $\mathbf{b ~ N i O} / \mathrm{Al}_{2} \mathrm{O}_{3}$ particle size distribution 
Table 1 Some worldwide databases in the area of neuroinformatics

\begin{tabular}{llllll}
\hline S.no & Parameters & Unit & \multicolumn{2}{l}{ Level } \\
\cline { 3 - 5 } & & & Minimum & Medium & Maximum \\
\hline 1 & Temperature & ${ }^{\circ} \mathrm{C}$ & 800 & 900 & 1000 \\
2 & $\begin{array}{c}\text { Flow rate of } \\
\text { precursor } \\
\text { gas }\end{array}$ & $\mathrm{ml} \mathrm{min}^{-1}$ & 100 & 140 & 180 \\
3 & Process time & $\min$ & 15 & 25 & 35 \\
\hline
\end{tabular}

carbon inclusions in the sample were equal to the well-aligned CNTs.

(iii) If the process time was less than 15 min, the defect was found in the outer ring of CNTs due to insufficient reaction time. If the process time was more than $35 \mathrm{~min}$, amorphous carbon and defective structure were found.

It was very difficult to study the effect of CVD process parameters on the diameter distribution of CNTs if misaligned CNTs were found in larger number. Hence, the above points were discussed to fix the working limit that decides the good surface morphology of CNTs.

All the above-listed limitations and conditions were considered and the feasible working limits were selected to conduct the experiments without any difficulties to attain the desired objective. The working range of CVD parameters employed in this investigation is presented in Table 1.

\section{Catalyst Preparation}

The $\mathrm{NiO} / \mathrm{Al}_{2} \mathrm{O}_{3}$ catalyst was prepared by impregnating $1 \mathrm{~g}$ of fumed alumina nanoparticles with $0.245 \mathrm{~g}$ of $\mathrm{Ni}\left(\mathrm{NO}_{3}\right)$. $6 \mathrm{H}_{2} \mathrm{O}$ in $30 \mathrm{ml}$ of methanol solution. The impregnation typically lasted for $1 \mathrm{~h}$ at room temperature using a magnetic stirrer. The methanol solution was removed via rotary evaporator and obtained material was then heated at $150{ }^{\circ} \mathrm{C}$ overnight followed by grinding into a fine powder. This resulting product, $[11,25]$ denoted as $\mathrm{NiO} / \mathrm{Al}$ was used as a catalyst in this investigation.

\section{Synthesize MWCNTs}

The MWCNTs were synthesized at atmospheric pressure in a quartz tube reactor (diameter: $70 \mathrm{~mm}$, length: $300 \mathrm{~mm}$ ) by thermal chemical vapor deposition machine. The synthesis of MWCNTs was carried out as per the conditions prescribed by the Table 1 .

In these experimental conditions, the catalyst loading $\left(10 \mathrm{mg}\right.$ ) and carrier gas (Argon: $100 \mathrm{ml} \mathrm{min}^{-1}$ ) flow rate were kept constant. The catalyst was loaded into the center of the hot zone reactor and then argon gas was supplied $\left(100 \mathrm{ml} \mathrm{min}^{-1}\right)$ until the attainment of required reaction temperature. Then argon was stopped and the carbon precursor gas (acetylene) was supplied to the reaction zone according to the process time. Later acetylene was replaced with argon until the furnace reaches room temperature. The carbon samples along with catalyst particles were collected from the quartz tube for the purification process to remove amorphous carbon and catalyst particles [11].

\section{Purify MWCNTs}

After the CVD process, the samples were purified by three steps (a) acid treatment (b) sonication and (c) filtered in sintering ceramic crucible. In the acid treatment step, the samples (derived from CVD process) were stirred in concentrated nitric acid for $3 \mathrm{~h}$ at room temperature. During this process of acid treatment carbon diffused samples, metal content was dissolved in the acid, but CNTs withstood the strong acid attack. After acid treatment, the metals (Catalysts particle) and amorphous carbon products were dissolved in acid solution due to the acid attack. The crystalline carbon products were free from catalyst [26-28] materials. The crystalline carbon samples were suspended in ethanol solution and sonicated for $10 \mathrm{~min}$. In this process, the agglomerated particles separated and MWCNTs were filtered in sintering ceramic crucible and carbon products were dried in a furnace.

\section{Characterize MWCNTs}

The MWCNTs synthesized by the CVD process were characterized by the field emission scanning electron microscopy (FESEM) technique. The FESEM analysis was carried out to confirm the presence of a tubular structure in the carbon samples. Further, carbon samples were analyzed by high-resolution transmission electron microscopy (HRTEM) technique, to examine the number of walls present in MWCNTs and its structure. The diameter distribution of MWCNTs was measured by Image $\mathrm{J}$ analysis software. The MWCNTs were analyzed by Raman spectroscopy, to reveal the presence of $\mathrm{G}$ and $\mathrm{D}$ band in the corresponding spectra, mainly to infer structural information about the tubes [29]. Further, the MWCNTs were subjected to TGA/DTA analysis to find the thermal stability and purity of tubes. The MWCNTs samples were subjected to image and analytical techniques, to obtain optimized CVD parameters and to study their effect on structure, stability, and diameter of MWCNTs. 

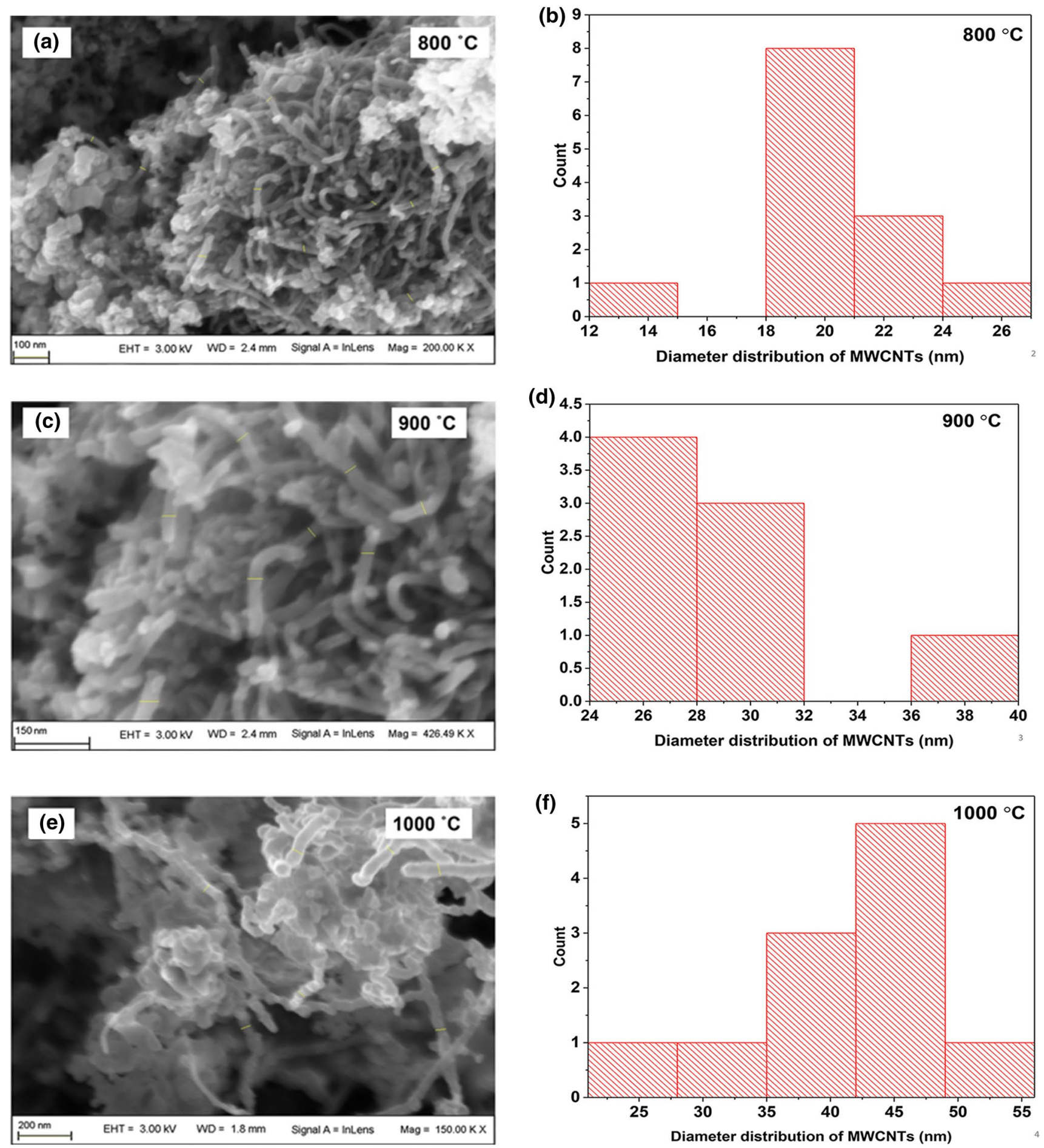

Fig. 2 FESEM image of MWCNTs grown at different reaction temperatures (a, $\mathbf{c}, \mathbf{e})$ and distribution of MWCNTs diameter as a function of reaction temperature $(\mathbf{b}, \mathbf{d}, \mathbf{f})$ 


\section{Results and discussion}

\section{Characterization of $\mathrm{NiO} / \mathrm{Al}$ catalyst}

For the controlled growth of CNTs, the size of the catalyst particles should be taken into consideration. The catalyst initiates the growth of CNTs and tube diameter governed by the size of the catalyst particle [30]. The Fig. 1a, b shows the $\mathrm{NiO} / \mathrm{Al}_{2} \mathrm{O}_{3}$ pristine structure, morphology and particle size distribution. The $\mathrm{NiO} / \mathrm{Al}_{2} \mathrm{O}_{3}$ catalyst particles have sphereshaped morphology and the particle size distribution ranges from 15 to $30 \mathrm{~nm}$ approximately. The particles are in circular shape and some catalyst particles are agglomerated even before the start of the experiment. So numerous factors may affect the diameter and distribution of CNTs.

\section{Effect of reaction temperature}

To study the effect of reaction temperature, it was varied from $800{ }^{\circ} \mathrm{C}$ to $1000{ }^{\circ} \mathrm{C}$ in steps of $100{ }^{\circ} \mathrm{C}$ but the precursor flow rate and process time were kept constant at $140 \mathrm{ml} \mathrm{min}^{-1}$ and $25 \mathrm{~min}$, respectively. The morphology of MWCNTs observed under FESEM and the diameter distribution of MWCNTs are depicted in Fig. 2.

The growth of MWCNTs at different reaction temperature shows the twisted and entangled tubular structure. At reaction temperature $800{ }^{\circ} \mathrm{C}$, the MWCNTs diameter distribution ranges from 15 to $28 \mathrm{~nm}$, at $900{ }^{\circ} \mathrm{C}$ MWCNTs diameter ranges from 25 to $40 \mathrm{~nm}$ and at $1000{ }^{\circ} \mathrm{C}$ diameter ranges from 28 to $53 \mathrm{~nm}$. From Fig. 2, it could be inferred that the reaction temperature is showing a directly proportional relationship with the mean diameter of the MWCNTs. The Fig. 3 shows the effect of reaction temperature on the mean diameter of MWCNTs. The enlargement of mean diameter of MWCNTs at higher temperatures is due to the sintering effect of catalyst particles results in small catalyst particle agglomerates to form larger particles [31].

The MWCNTs diameter distribution (15-24 nm) at reaction temperature $800{ }^{\circ} \mathrm{C}$ is relatively depended on the catalyst particles diameter distribution $(15-30 \mathrm{~nm})$ and it is clearly evident in Fig. 2a. The MWCNTs diameter distribution $(22-32 \mathrm{~nm})$ at $900{ }^{\circ} \mathrm{C}$ is quite higher compared to the catalyst particles distribution. Therefore, the agglomeration of catalyst particles starts from the reaction temperature above $800{ }^{\circ} \mathrm{C}$. It is understood that the increase in reaction temperature tends to increase the reduction temperature [32]; this reduction effect causes the catalyst particles to agglomerate and agglomerated particles result in a growth of larger diameter MWCNTs.

\section{Effect of the flow rate of the precursor gas}

To study the effect of precursor gas flow rate, it was varied from 100 to $180 \mathrm{ml} \mathrm{min}{ }^{-1}$ in steps of $40 \mathrm{ml} \mathrm{min}^{-1}$ but the reaction temperature and process time were kept constant at $900{ }^{\circ} \mathrm{C}$ and $25 \mathrm{~min}$, respectively. The morphology of MWCNTs observed under FESEM and the diameter distribution of MWCNTs are depicted in Fig. 4. The MWCNTs growth at a higher flow rate of precursor gas shows the deposition of amorphous carbon on the sidewalls of CNTs. At flow rate of precursor gas $100 \mathrm{ml} \mathrm{min}^{-1}$, the MWCNTs diameter distribution ranges from 14 to $22 \mathrm{~nm}$; at $140 \mathrm{ml} \mathrm{min}^{-1}$, from 25 to $40 \mathrm{~nm}$; and at $180 \mathrm{ml} \mathrm{min}{ }^{-1}$, from 25 to $52 \mathrm{~nm}$. From Fig. 4, it could be inferred that the flow rate of precursor gas is showing a directly proportional relationship with the mean diameter of the MWCNTs. Fig. 5 shows the effect of precursor flow rate on the mean diameter of MWCNTs.

The diffusion of carbon atoms towards the catalyst particles was low, at a low flow rate of acetylene gas [17]. The lack of diffusion retracts the dispersion of carbon atoms in the catalyst particles leading to decrease in diameter distribution of MWCNTs clearly evident in Fig. 4a. If the flow rate of acetylene gas is high, the diffusion of large amounts of carbon atoms towards the catalyst particle was high that tends to increase the dispersion rate. At high diffusion rate of carbon, the large amount carbon present in catalyst particle starts to reduce the activity of the catalyst, due to catalyst particles being covered by amorphous carbon, that increase the thickness of the MWCNTs [33] and it is clearly evident in Fig. 4e.

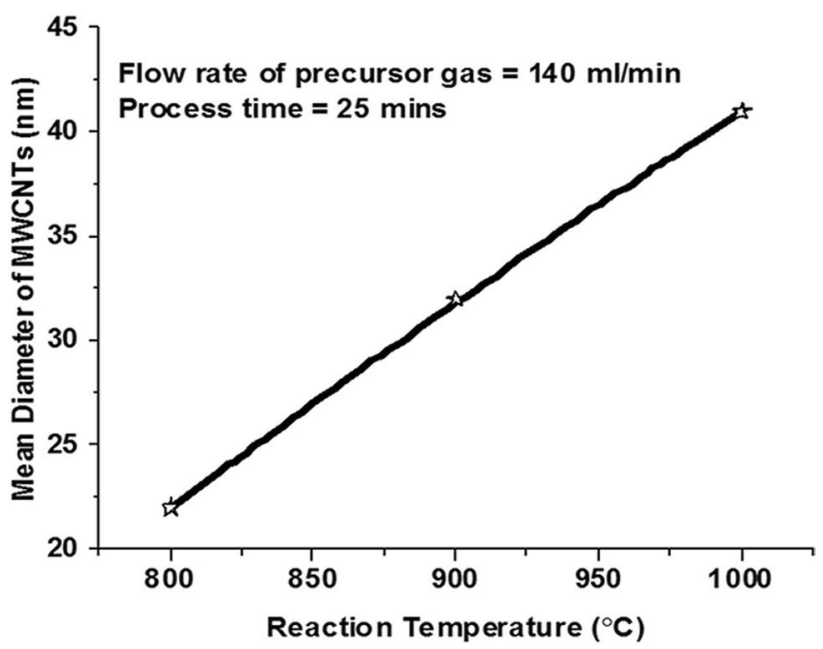

Fig. 3 Effect of reaction temperature on the mean diameter of MWCNTs 


\section{Effect of process time}

To study the effect of process time, it was varied from $15 \mathrm{~min}$ to $35 \mathrm{~min}$ in steps of $10 \mathrm{~min}$ but the reaction temperature and flow rate of precursor gas were kept constant at $900{ }^{\circ} \mathrm{C}$ and
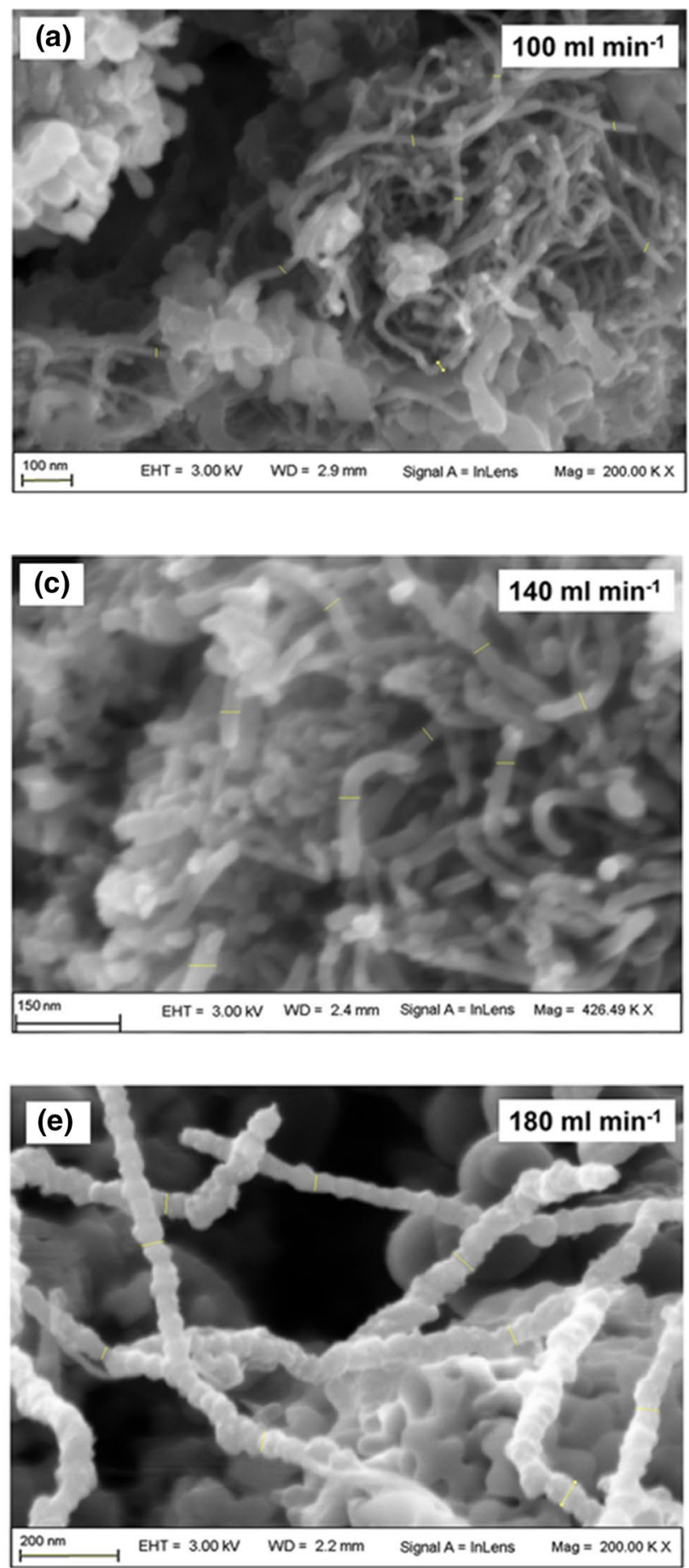

$140 \mathrm{ml} \mathrm{min}{ }^{-1}$, respectively. The morphology of MWCNTs observed under FESEM and the diameter distribution of MWCNTs are depicted in Fig. 6. The MWCNTs' growth at different processing time shows the bundles and well-aligned carbon structures. At a process time of $15 \mathrm{~min}$, the diameter (b) 4

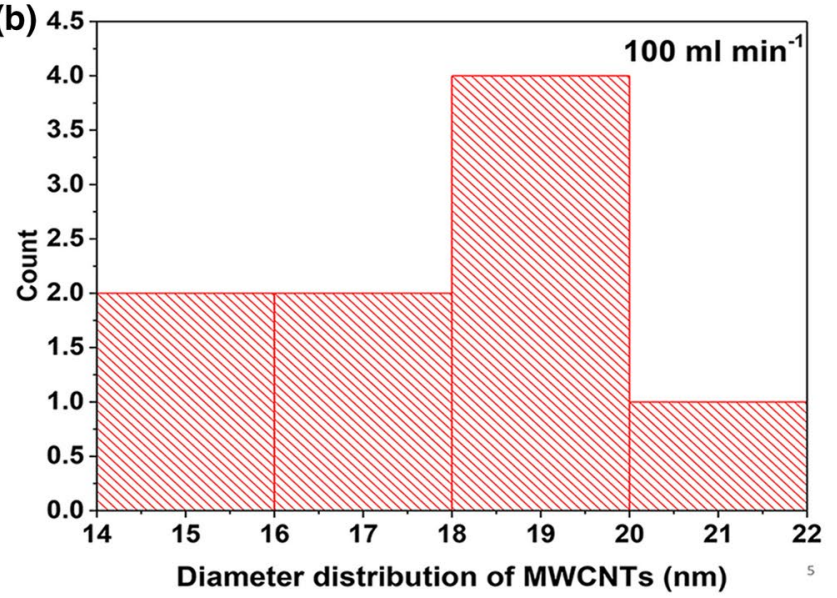

(d)
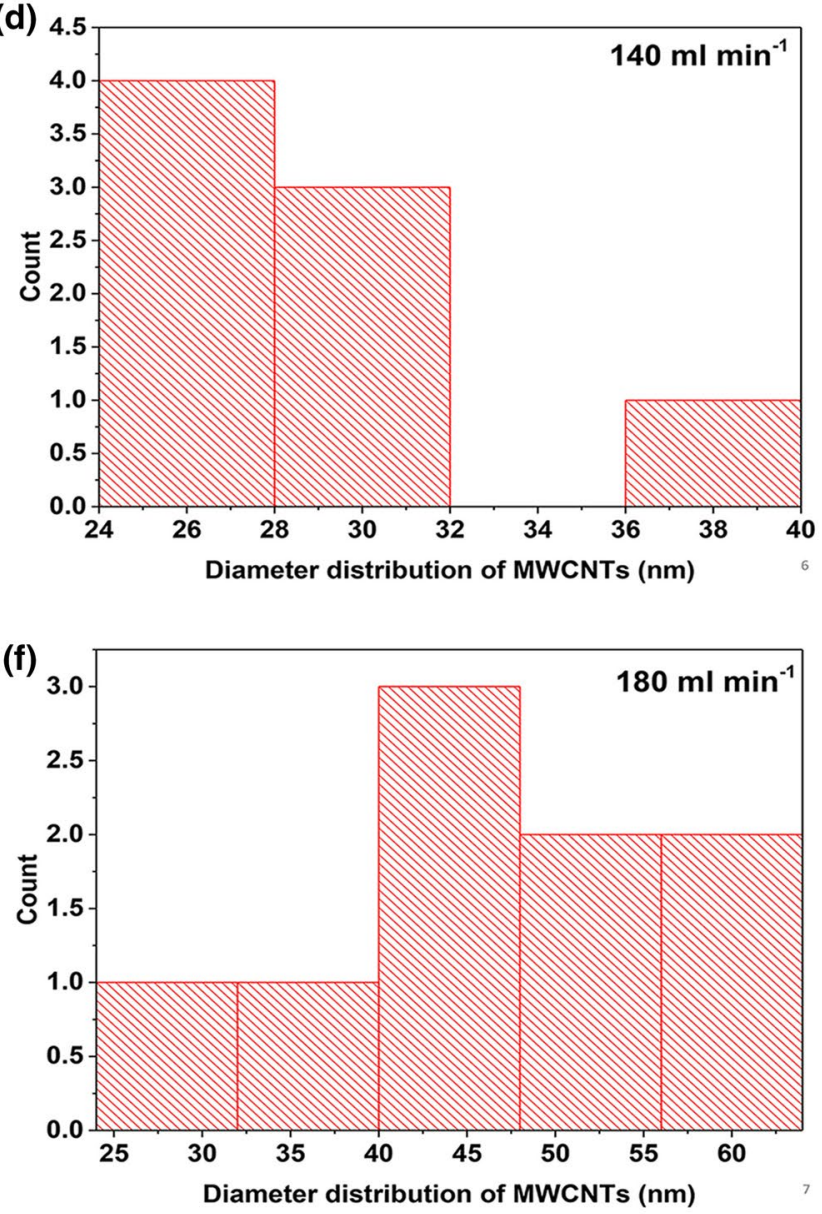

Fig. 4 FESEM image of MWCNTs grown at different flow rate of precursor gas (a, $\mathbf{c}, \mathbf{e})$ and distribution of MWCNTs diameter as a function of flow rate of precursor gas $(\mathbf{b}, \mathbf{d}, \mathbf{f})$ 
of MWCNTs ranges from $20 \mathrm{~nm}$ to $50 \mathrm{~nm}$, at the process time of $25 \mathrm{~min}$, the diameter ranges from 25 to $40 \mathrm{~nm}$ and at process time of $35 \mathrm{~min}$, the diameter ranges from 10 to $30 \mathrm{~nm}$. From Fig. 6, it could be inferred that the process time is showing the inversely proportional relationship with the mean diameter of the MWCNTs. Fig. 7 shows the effect of process time on the mean diameter of MWCNTs.

The mean diameter of the MWCNTs decreases, when processing time increases. This effect can be explained by two hypotheses,

(i) Nagaraju.et al. [15] reported that higher reaction time favors better results in acetylene-to-nanotube conversion compared to lower reaction time and higher process time which tends to allow appropriate time to the activity of the catalyst.

(ii) Navas et al. [34] confirmed that smaller diameter tube can form at longer process time. Basically, CNTs with a smaller diameter are observed at intermediate temperature and precursor pressure, which also corresponds to the conditions leading to the highest proportions of small diameters. The small diameter CNTs generally form later than the larger ones, but their onset can be accelerated by increasing the temperature or the precursor gas pressure.

Though the sample that was grown at 35 min composed of both larger (more than $25 \mathrm{~nm}$ ) and smaller diameter MWCNTs, the smaller diameter MWCNTs are predominantly higher and it is clearly evident from Fig. $6 \mathrm{f}$.

\section{Optimization of process parameters to attain controlled diameter distribution and defectless structure}

The main aim of this investigation is to study the effect of CVD process parameters on the diameter of MWCNTs and to optimize appropriate processing conditions that result defectless structure with the smallest diameter.

To attain these conditions, HRTEM, Raman spectroscopy, and thermo gravimetric analysis/differential thermogravimetry were utilized to examine the MWCNTs. From the FESEM analysis, it is found that the MWCNTs synthesized at 15-mins and 35-mins process time (Fig. 6) yielded controlled diameter distribution and defectless side wall of MWCNTs.

\section{HRTEM analysis}

Figure 8 presents HRTEM images of the MWCNTs grown at $35 \mathrm{~min}$ and $15 \mathrm{~min}$ processing time, respectively. Compared to 15 min process time, the growth of MWCNTs at 35 min process time shows defect-free, well-aligned structure and controlled diameter distribution. The little scatter in the diameter distributions observed (Fig. 6) in both the process conditions is due to starting catalyst particles which were agglomerated in nature even before the start of the experiment. The MWCNTs tip growth mode was clearly evident in Fig. 8c indicated by an arrow mark. The MWCNTs starts reacting with the electron source that is indicated by the circle. The reaction of MWCNTs with source electrons while conducting HRTEM analysis shows the stability of the carbon that presents in Fig. 8c.

At higher temperature, acetylene decomposition tends to reduce when it reacts with the catalyst to form a solid carbon [30]; This also may be another reason for attaining controlled diameter distribution at longer process time.

\section{Raman spectroscopy analysis}

Figure 9 shows the Raman spectrum of MWCNTs grown at 35 and 15 min process time. The peaks observed at 1370 , $1385 \mathrm{~cm}^{-1}$ correspond to the D (defective) band of MWCNTs synthesized at 15 and $35 \mathrm{~min}$, respectively. The peaks at $1570,1585 \mathrm{~cm}^{-1}$ are characteristics peaks due to the scattering of $\mathrm{G}$ band (graphitic mode) of MWCNTs synthesized at 15 and $35 \mathrm{~min}$. The $680 \mathrm{~cm}^{-1}$ Raman shift peak was observed in MWCNTs sample synthesized at $35 \mathrm{~min}$ process time, which is near to the RBM mode. The presence of RBM peak is due to the shorter diameter of MWCNTs. The intensity ratio between $\mathrm{D}$ and $\mathrm{G}$ band peaks is used to evaluate the graphitization degree of CNTs. The lower value of $\mathrm{I}_{\mathrm{D}} / \mathrm{I}_{\mathrm{G}}$ consequently relates to the high quality of MWCNTs and comparatively higher value than this corresponds to the poor structural quality, which has structural disorder [35]. The MWCNTs grown at $35 \mathrm{~min}$ process time show a higher degree of crystallinity compared to MWCNTs grown at 15 min process time. The observed $I_{\mathrm{D}} / I_{\mathrm{G}}$ values are having good agreement with the HRTEM analysis.

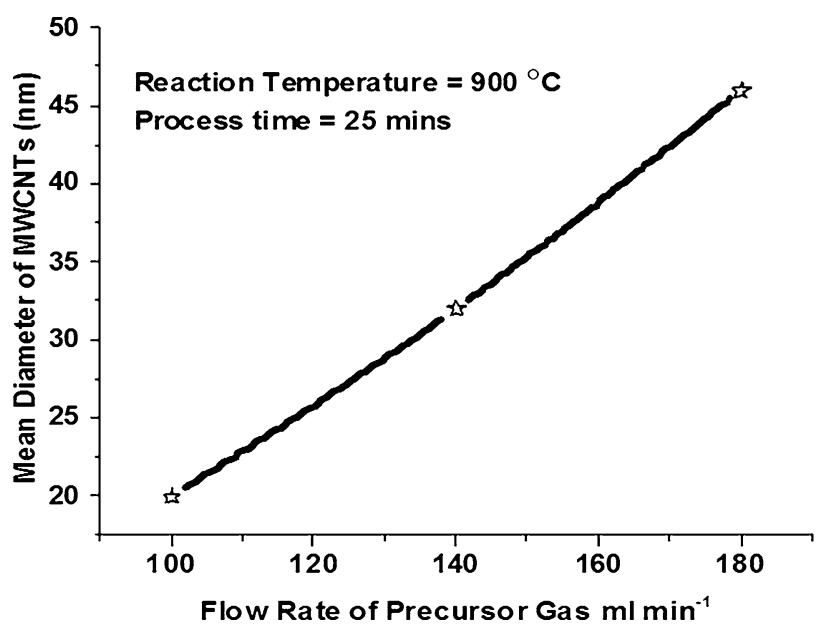

Fig. 5 Effect of precursor gas flow rate on mean diameter of MWCNTs 


\section{Thermogravimetric analysis (TGA)}

Thermogravimetry was used to evaluate the thermal stability and purity of CNTs sample. Figure 10 shows the TG and DTA curve of MWCNTs grown at 15-mins and 35-mins process time. The nature of an exothermic peak is expected to occur
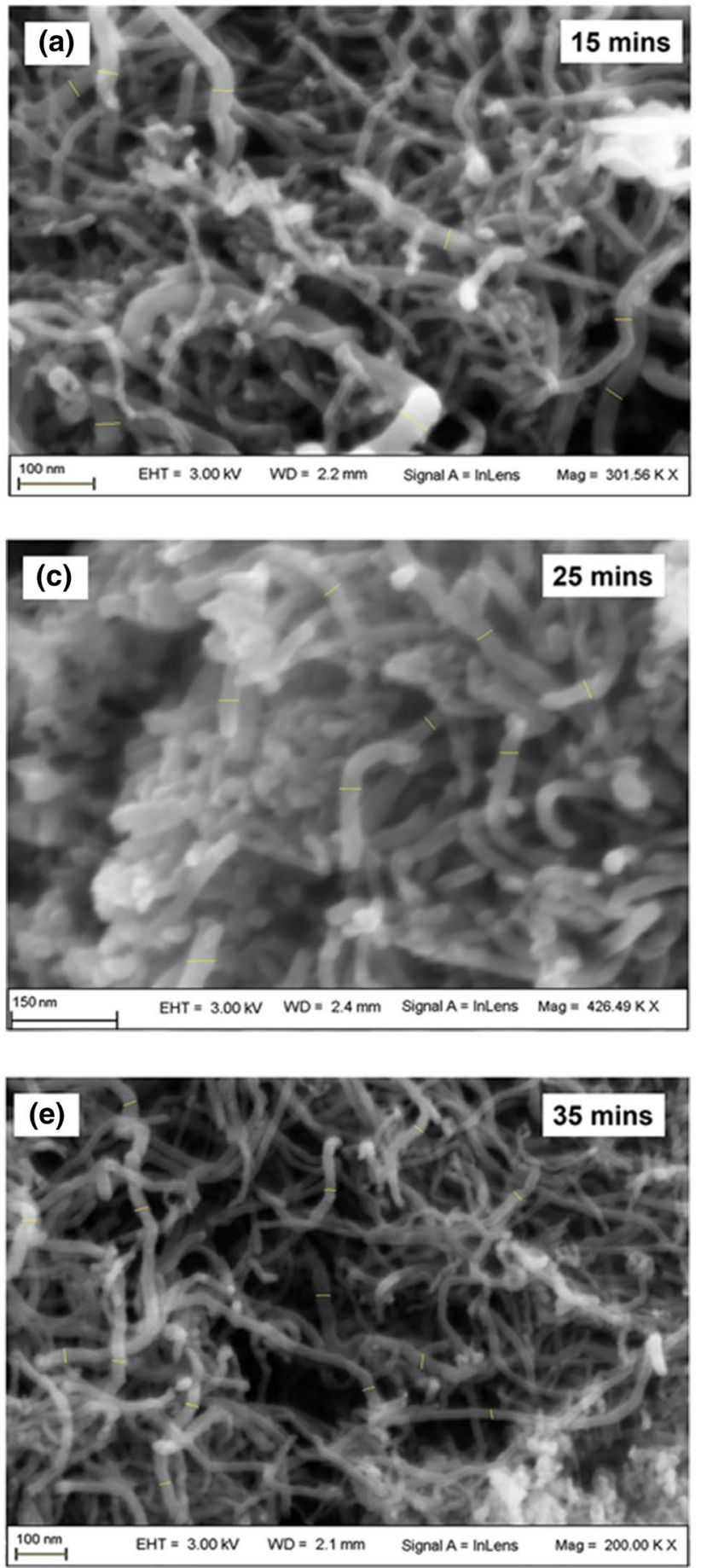

from the oxidation of the sample [36]. Hence, the largest exothermic peak in the DTA curve is near $680{ }^{\circ} \mathrm{C}$ (green color), which indicates the combustion of MWCNTs. The peak at $300{ }^{\circ} \mathrm{C}$ in the DTA (green color) curve is considered to be the oxidation of amorphous carbon. The TGA/DTA graph for MWCNTs grown at 15 min process time also depicted in
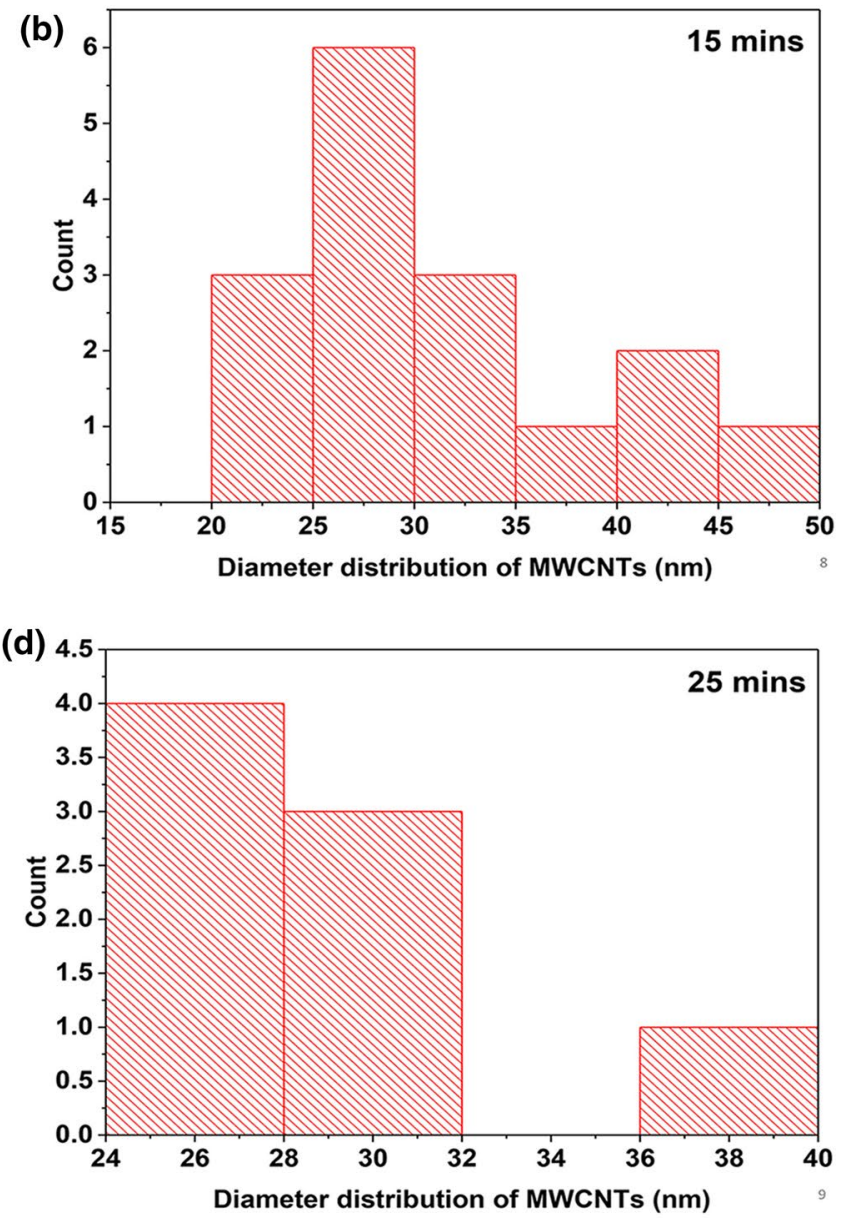

(f)

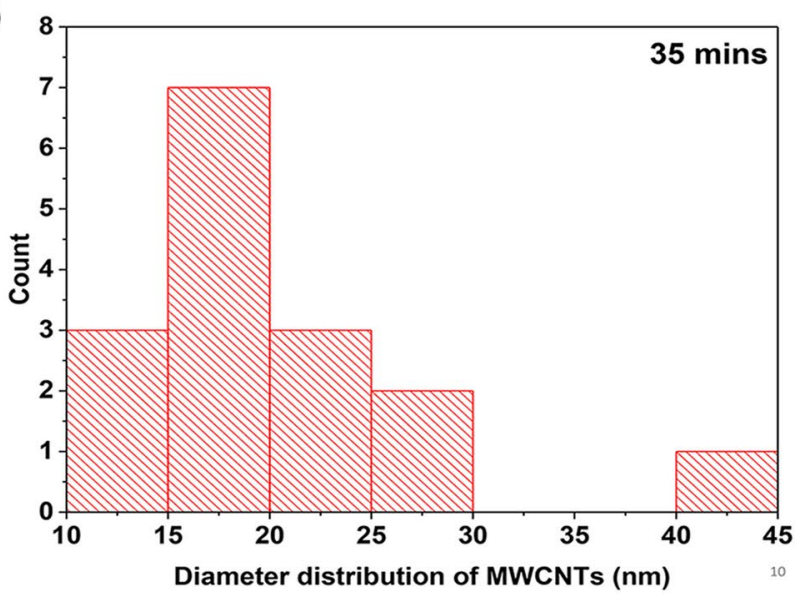

Fig. 6 FESEM image of MWCNTs grown at different processing time (a, $\mathbf{c}, \mathbf{e})$ and distribution of MWCNTs diameter as a function of processing time $(\mathbf{b}, \mathbf{d}, \mathbf{f})$ 


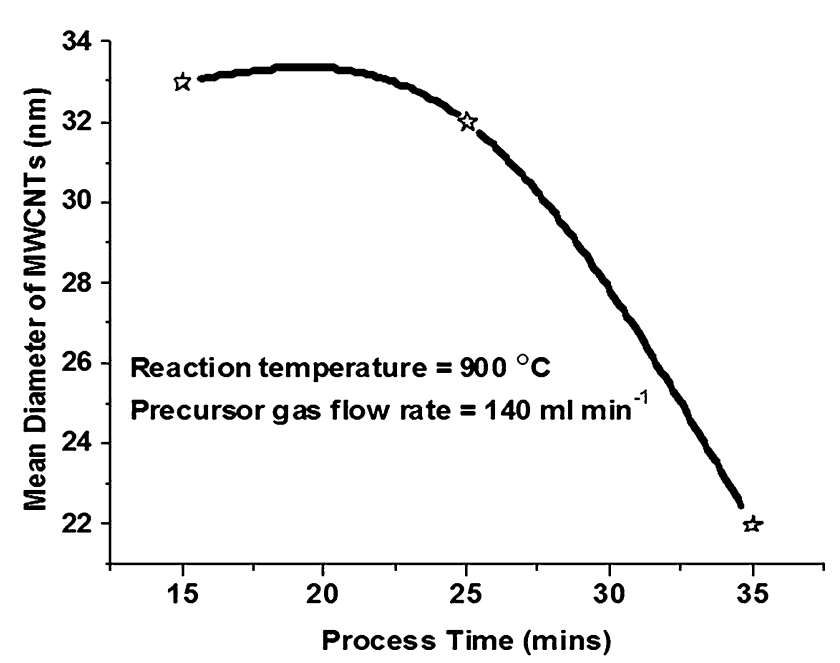

Fig. 7 Effect of processing time on mean diameter of MWCNTs

Fig. 10. The exothermic reaction peak in DTA curve (violet color) around $150{ }^{\circ} \mathrm{C}$ occurred due to the oxidation of amorphous carbon. Significant weight loss was also observed in TGA curve (violet color), which reveals the non-stability of the sample above $400{ }^{\circ} \mathrm{C}$. The TGA analysis for MWCNTs

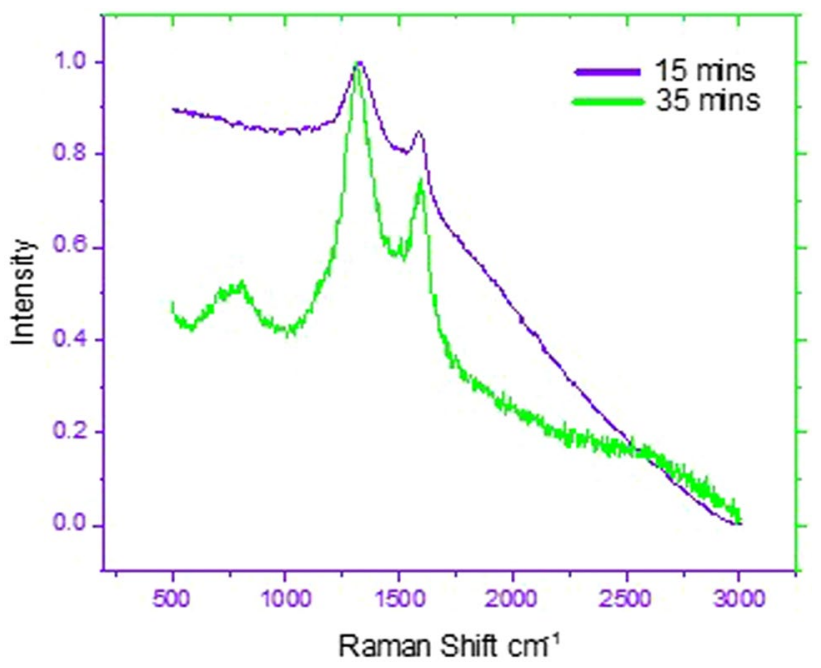

Fig. 9 Raman spectra of carbon materials synthesized at 15 and 35-mins process time

synthesized at $15 \mathrm{~min}$ and $35 \mathrm{~min}$ show a weight loss of $25 \%$ and $2 \%$, respectively. From the TGA/DTA curves, it is understood that the MWCNTs grown at 35 min process time show
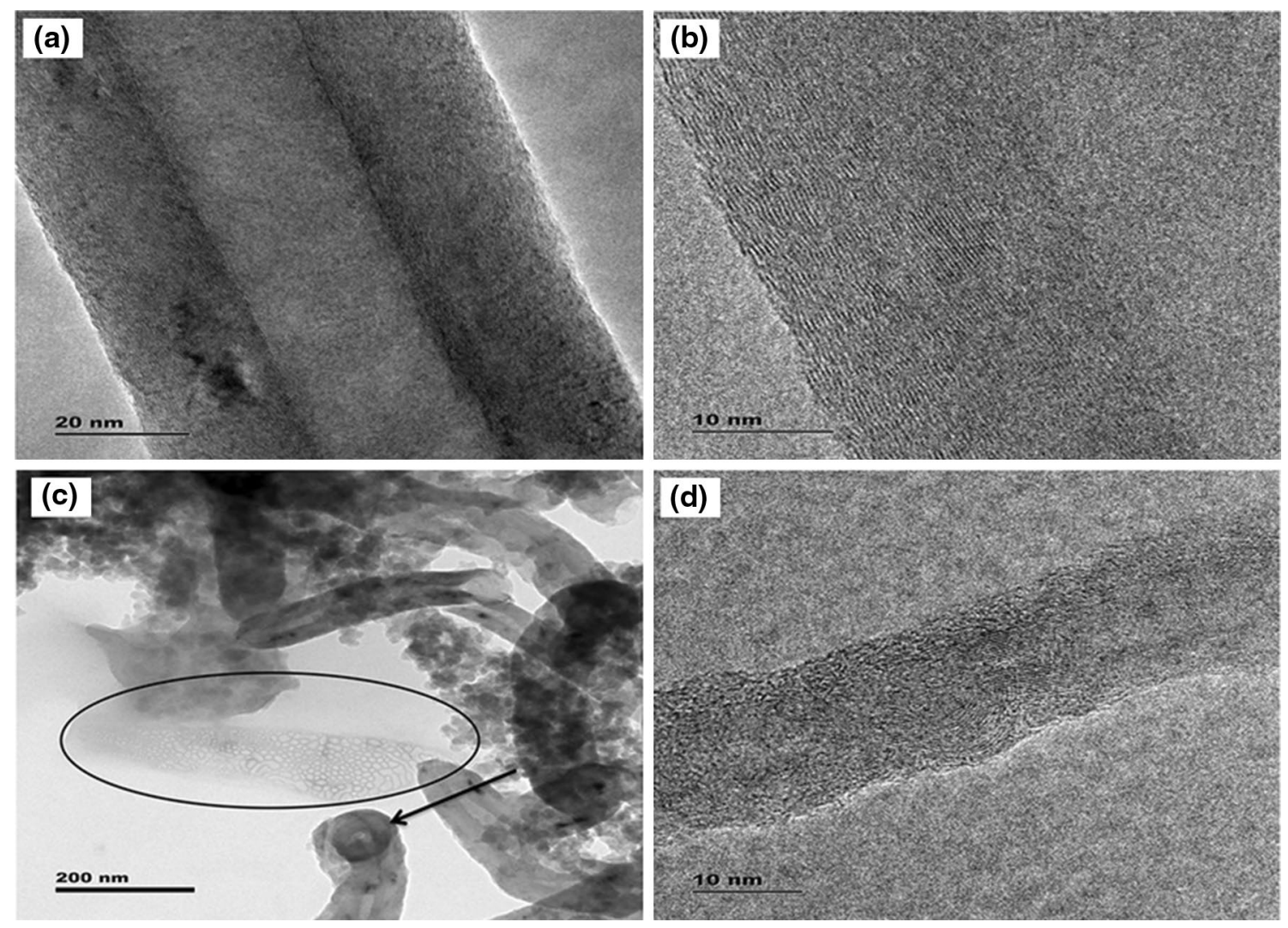

Fig. 8 HRTEM image a MWCNTs growth at 35 mins process time, $\mathbf{b}$ higher resolution image of a, $\mathbf{c}$ MWCNTs growth at 15 mins process time, d higher resolution image of $\mathbf{c}$ 
Fig. 10 TGA/DTA profile of MWCNTs grown at 15-mins and 35-mins processing time

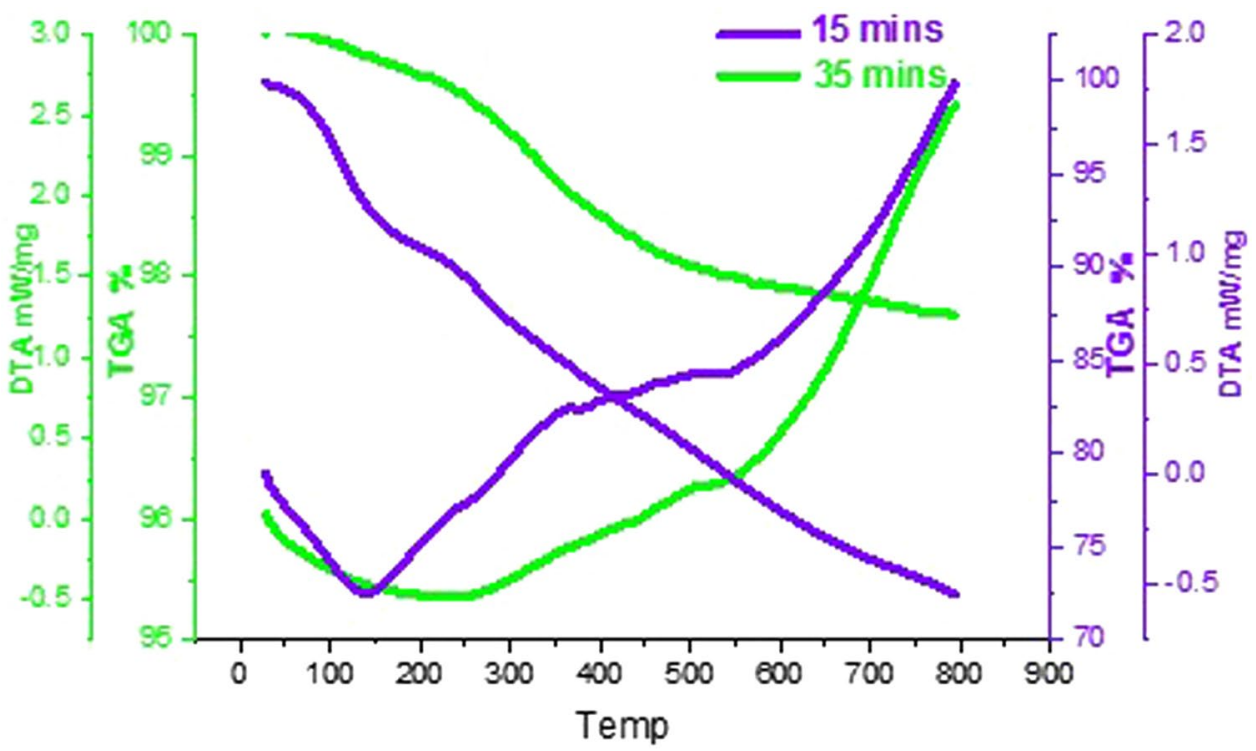

higher oxidative stability and form CNTs with well-aligned graphitic structure.

\section{Conclusions}

In this paper, the effect of CVD process parameters on the diameter of MWCNTs has been analyzed in detail. From this investigation, the following important conclusions are drawn.

(i) The reaction temperature is showing directly proportional relationship with the mean diameter of MWCNTs. If the reaction temperature increases, the diameter of MWCNTs increases and vice versa. At higher reaction temperature, the catalyst particles get agglomerated and this causes an increase in diameter of MWCNTs.

(ii) The precursor flow rate is showing directly proportional relationship with the mean diameter of MWCNTs. If the precursor flow rate increases, the diameter of MWCNTs increases and vice versa. At a higher flow rate of precursor gas (carbon gas), concentrate carbon particles increases and this lead to the faster rate of carbon diffusion and subsequently increases the diameter of MWCNTs.

(iii) The process (reaction) time is showing an inversely proportional relationship with the mean diameter of MWCNTs. If the process time increases, the diameter of MWCNTs decreases and vice versa. At longer process time, the onset growth of smaller diameter CNTs was accelerated by the higher reaction temperature and this facilitates the growth of smaller diameter of MWCNTs. (iv) From the Raman peak analysis and TGA/DTA test results, it is concluded that MWCNTs synthesized at 35 min process time, has higher graphitization and higher oxidation temperature.

(v) From this investigation, it is found that reaction temperature of $900{ }^{\circ} \mathrm{C}$, precursor gas flow rate $140 \mathrm{ml} \mathrm{min}^{-1}$ and process time of $35 \mathrm{~min}$ yielded defectless, wellaligned MWCNTs compared to all other experimental conditions.

Acknowledgements The first author gratefully acknowledges the financial support provided by M/s. VB Ceramics Consultants (VBCC), Nehru Nagar Industrial Estate, Kottivakkam, Chennai-600041, India through VBCRF (VB CERAMICS RESEARCH FELLOWSHIP). The authors are grateful to The Director, Naval Materials Research Laboratory (NMRL), DRDO, Ambernath, Maharastra for granting permission to utilize FESEM facility to characterize MWCNTs.

\section{Compliance with ethical standards}

Conflict of interest The authors declare that they have no conflict of interest.

Open Access This article is distributed under the terms of the Creative Commons Attribution 4.0 International License (http://creativeco mmons.org/licenses/by/4.0/), which permits unrestricted use, distribution, and reproduction in any medium, provided you give appropriate credit to the original author(s) and the source, provide a link to the Creative Commons license, and indicate if changes were made.

\section{References}

1. Iijima, S.: Helical micro tubules of graphitic carbon. Nature 354, $56(1991)$ 
2. Atthipalli, G., Epur, R., Kumta, P.N., Allen, B.L., Tang, Y., Star, A., Gray, J.L.: The effect of temperature on the growth of carbon nanotubes on copper foil using a nickel thin film as catalyst. Thin Solid Films (2011). https://doi.org/10.1016/j.tsf.2011.02.046

3. Peng, B., Locascio, M., Zapol, P., Li, S., Mielke, S.L., Schatz, G.C., Espinosa, H.D.: Measurements of near-ultimate strength for multiwalled carbon nanotubes and irradiation-induced crosslinking improvements. Nat. Nano. 3, 626-631 (2008). https://doi. org/10.1038/nnano.2008.211

4. De Volder, M.F.L., Tawfick, S.H., Baughman, R.H., Hart, A.J.: Carbon nanotubes: present and future commercial applications. Science (2013). https://doi.org/10.1126/science.1222453

5. Hutchison, J.L., Kiselev, N.A., Krinichnaya, E.P., Krestinin, A.V., Loutfy, R.O., Morawsky, A.P., Muradyan, V.E., Obraztsova, E.D., Sloan, J., Terekhov, S.V., Zakharov, D.N.: Double-walled carbon nanotubes fabricated by a hydrogen arc discharge method. Carbon N. Y. (2001). https://doi.org/10.1016/S0008-6223(00)00187-1

6. Chrzanowska, J., Hoffman, J., Małolepszy, A., Mazurkiewicz, M., Kowalewski, T.A., Szymanski, Z., Stobinski, L.: Synthesis of carbon nanotubes by the laser ablation method: effect of laser wavelength. Phys. Status solidi. (2015). https://doi.org/10.1002/ pssb.201451614

7. Kumar, M., Ando, Y.: Controlling the diameter distribution of carbon nanotubes grown from camphor on a zeolite support. Carbon N. Y. (2005). https://doi.org/10.1016/j.carbon.2004.10.014

8. Yousefi, A.T., Mahmood, M.R., Ikeda, S.: Growth of welloriented VACNTs using thermal chemical vapor deposition method. AIP Conf. Proc. 020038, 020038 (2016). https://doi. org/10.1063/1.4948856

9. Kumar, M., Ando, Y.: Chemical vapor deposition of carbon nanotubes: a review on growth mechanism and mass production. J. Nanosci. Nanotechnol. (2010). https://doi.org/10.1166/ jnn.2010.2939

10. MacKenzie, K.J., Dunens, O.M., Harris, A.T.: An updated review of synthesis parameters and growth mechanisms for carbon nanotubes in fluidized beds. Ind. Eng. Chem. Res. (2010). https://doi. org/10.1021/ie9019787

11. Kong, J., Cassell, A.M., Dai, H.: Chemical vapor deposition of methane for single-walled carbon nanotubes. Chem. Phys. Lett. 21, 10 (1998). https://doi.org/10.1016/s0009-2614(98)00745-3

12. Zhang, C., van Duin, A.C.T., Seo, J.W., Seveno, D.: Weakening effect of nickel catalyst particles on the mechanical strength of the carbon nanotube/carbon fiber junction. Carbon N. Y. (2017). https ://doi.org/10.1016/j.carbon.2017.01.042

13. He, C.N., Zhao, N.Q., Shi, C.S., Song, S.Z.: Fabrication of carbon nanomaterials by chemical vapor deposition. J. Alloys Compd. 484, 6-11 (2009). https://doi.org/10.1016/j.jallcom.2009.04.088

14. Yemini, R., Itzhak, A., Gofer, Y., Sharabani, T., Drela, M., Nessim, G.D.: Nickel overlayers modify precursor gases to pattern forests of carbon nanotubes. J. Phys. Chem. C (2017). https://doi. org/10.1021/acs.jpcc.7b00801

15. Nagaraju, N., Fonseca, A., Konya, Z., Nagy, J.B.: Alumina and silica supported metal catalysts for the production of carbon nanotubes. J. Mol. Catal. A Chem. (2002). https://doi.org/10.1016/ s1381-1169(01)00375-2

16. Satishkumar, B.C., Govindaraj, A., Sen, R., Rao, C.N.R.: Singlewalled nanotubes by the pyrolysis of acetylene-organometallic mixtures. Chem. Phys. Lett. (1998). https://doi.org/10.1016/s0009 -2614(98)00727-1

17. Cao, Z., Sun, Z., Guo, P., Chen, Y.: Effect of acetylene flow rate on morphology and structure of carbon nanotube thick films grown by thermal chemical vapor deposition. Front. Mater. Sci. China. (2007). https://doi.org/10.1007/s11706-007-0017-x

18. Tewari, A., Sharma, S.C.: Effect of different carrier gases and their flow rates on the growth of carbon nanotubes. Phys. Plasmas. (2015). https://doi.org/10.1063/1.4916057
19. Shukrullah, S., Mohamed, N.M., Shaharun, M.S., Naz, M.Y.: Effect of ethylene flow rate and CVD process time on diameter distribution of MWCNTs. Mater. Manuf. Process. (2016). https ://doi.org/10.1080/10426914.2015.1090588

20. He, C.N., Zhao, N.Q., Shi, C.S., Song, S.Z.: An approach for obtaining the structural diversity of multi-walled carbon nanotubes on Ni/Al catalyst with low Ni content. J. Alloys Compd. (2010). https://doi.org/10.1016/j.jallcom.2009.09.067

21. Inami, N., Ambri Mohamed, M., Shikoh, E., Fujiwara, A.: Synthesis-condition dependence of carbon nanotube growth by alcohol catalytic chemical vapor deposition method. Sci. Technol. Adv. Mater. (2007). https://doi.org/10.1016/j.stam.2007.02.009

22. Lolli, G., Zhang, L., Balzano, L., Sakulchaicharoen, N., Tan, Y., Resasco, D.E.: Tailoring (n, m) structure of single-walled carbon nanotubes by modifying reaction conditions and the nature of the support of CoMo catalysts. J. Phys. Chem. B. (2006). https://doi. org/10.1021/jp056095e

23. Mubarak, N.M., Yusof, F., Alkhatib, M.F.: The production of carbon nanotubes using two-stage chemical vapor deposition and their potential use in protein purification. Chem. Eng. J. 168, 461-469 (2011). https://doi.org/10.1016/j.cej.2011.01.045

24. Allaedini, G., Tasirin, S.M., Aminayi, P.: Synthesis of Fe-Ni-Ce trimetallic catalyst nanoparticles via impregnation and co-precipitation and their application to dye degradation. Chem. Pap. (2015). https://doi.org/10.1515/chempap-2015-0190

25. Makris, T.D., Giorgi, L., Giorgi, R., Lisi, N., Salernitano, E.: CNT growth on alumina supported nickel catalyst by thermal CVD. Diam. Relat. Mater. (2005). https://doi.org/10.1016/j.diamo nd.2004.11.001

26. Guan, B.H., Ramli, I., Yahya, N., Pah, L.K.: Purification of carbon nanotubes synthesized by catalytic decomposition of methane using bimetallic Fe-Co catalysts supported on $\mathrm{MgO}$. IOP Conf. Ser. Mater. Sci. Eng. (2011). https://doi.org/10.1088/1757899x/17/1/012025

27. Zheng, J., Bao, R., Yi, J.H., Yang, P.: Microwave purification of multi-wall carbon nanotubes in gas phase. Diam. Relat. Mater. (2016). https://doi.org/10.1016/j.diamond.2016.06.006

28. Ling, X., Wei, Y., Zou, L., Xu, S.: The effect of different order of purification treatments on the purity of multiwalled carbon nanotubes. Appl. Surf. Sci. (2013). https://doi.org/10.1016/j.apsus c.2013.03.056

29. Lehman, J.H., Terrones, M., Meunier, V., Mansfield, E., Hurst, K.E.: Evaluating the characteristics of multiwall carbon. Carbon N. Y. (2011). https://doi.org/10.1016/j.carbon.2011.03.028

30. Kim, M.S., Rodriguez, N.M., Baker, R.T.K.: The interaction of hydrocarbons with coppernickel and nickel in the formation of carbon filaments. J. Catal. (1991). https://doi.org/10.1016/00219517(91)90323-v

31. Kukovitsky, E.F., Lvov, S.G., Sainov, N.A., Shustov, V.A., Chernozatonskii, L.A.: Correlation between metal catalyst particle size and carbon nanotube growth. Chem Phys Lett. (2002). https://doi. org/10.1016/s0009-2614(02)00283-x

32. He, C.N., Zhao, N.Q., Du, X.W., Shi, C.S., Li, J.J., He, F.: Characterization of bamboo-shaped CNTs prepared using depositionprecipitation catalyst. Mater. Sci. Eng. A. (2008). https://doi. org/10.1016/j.msea.2007.06.048

33. Tripathi, N., Mishra, P., Harsh, H., Islam, S.S.: Fine-tuning control on CNT diameter distribution, length and density using thermal CVD growth at atmospheric pressure: an in-depth analysis on the role of flow rate and flow duration of acetylene $(\mathrm{C} 2 \mathrm{H} 2)$ gas. Appl. Nanosci. (2015). https://doi.org/10.1007/s13204-013-0288-8

34. Navas, H., Picher, M., Andrieux-ledier, A., Michel, T., Kozawa, A., Maruyama, T., Anglaret, E., Loiseau, A., Jourdain, V.: Unveiling the evolutions of nanotube. ACS Nano. (2017). https://doi. org/10.1021/acsnano.7b00077 
35. Rajavel, K., Dinesh, M., Saranya, R., Rajendra kumar, R.T.: Enhanced vacuum sensing performance of multiwalled carbon nanotubes: role of defects and carboxyl functionalization. RSC Adv. (2014). https://doi.org/10.1039/x0xx00000x

36. Kumar, K., Pandey, A.C., Parkash, O.: Photon avalanche upconversion and pump power studies in $\mathrm{LaF} 3$ : $\mathrm{Er} 3+/ \mathrm{Yb} 3+$ phosphor. Appl Phys B Lasers O (2014). https://doi.org/10.1007/ s00340-011-4673-2

Publisher's Note Springer Nature remains neutral with regard to jurisdictional claims in published maps and institutional affiliations. 